\title{
Modelo predictor de ingreso hospitalario a la llegada al servicio de Urgencias
}

\section{Prediction model for in-hospital admission in patients arriving in the Emergency Department}

\author{
C.M. Elvira Martínez ${ }^{1}$, C. Fernández ${ }^{2,4}$, J. González del Castillo ${ }^{3}$, \\ J.J. González-Armengol ${ }^{3}$, P. Villarroel ${ }^{3}$, F.J. Martín-Sánchez ${ }^{3}$
}

\section{RESUMEN}

Fundamento. Desarrollar un modelo de predicción de ingreso hospitalario a la llegada del paciente al servicio de Urgencias, con el fin de conocer la necesidad de camas hospitalarias casi a tiempo real, y así prever los recursos asistenciales necesarios de forma precoz.

Material y métodos. Estudio observacional de cohorte prospectivo. Se incluyeron todos los pacientes consecutivos filiados para el triaje entre las 8-22 horas del servicio de Urgencias de un hospital terciario durante un mes. Se analizaron 7 variables a la llegada del paciente, que pudieran influir en el ingreso: edad, sexo, nivel de gravedad según el triaje, ubicación inicial, diagnóstico de entrada, solicitud de prueba complementaria y prescripción de medicación. Se realizó un estudio multivariable según regresión logística.

Resultados. Se incluyeron 2.476 episodios de los que 114 $(4,6 \%)$ ingresaron. Se asociaron de forma significativa: edad $>65$ años (Odds ratio $[\mathrm{OR}]=2,1$, intervalo de confianza [IC] $95 \%, 1,3-3,2 ; \mathrm{p}=0,001$ ); sexo masculino (OR=1,6, IC 95\%,1,1-2,4; $\mathrm{p}=0,020)$. Diagnóstico de entrada disnea: (OR=5,2, IC $95 \%$, $2,8-9,7 ; \mathrm{p}<0,0001)$; dolor abdominal (OR=4,7, IC $95 \%, 2,7-8,3$; $\mathrm{p}<0,0001)$; ubicación inicial en sala de agudos $(\mathrm{OR}=8,9$, IC $95 \%, 5,4-14,9 ; \mathrm{p}<0,0001)$; solicitud de pruebas complementarias (OR=1,1, IC95\%,0,9-1,3; $\mathrm{p}=0,064)$ y prescripción de tratamiento $(\mathrm{OR}=2,6$, IC $95 \%, 1,6-4,2 ; \mathrm{p}=<0,0001)$. Con dichas variables se diseñó un modelo matemático que tenía una sensibilidad del $76 \%$ y una especificidad del $82 \%$ (área bajo la curva es de 0,85 [IC $95 \% 0,81-0,88 ; \mathrm{p}<0,001]$ ).

Conclusiones. El modelo de predicción de ingreso es una herramienta que puede ser de utilidad a la hora de prever la necesidad del recurso cama hospitalaria a la llegada del paciente al servicio de Urgencias.

Palabra clave. Triaje. Urgencias. Ingreso. Saturación.

\begin{abstract}
Background. To develop a prediction model for in-hospital admission to provide an almost "real time" determination of hospital beds needed, so as to predict the resources required as early as possible.
\end{abstract}

Material and methods. A prospective observational study in the emergency department of a university hospital. We included all consecutive patients between 8.00-22.00 hours during one month. We analyzed 7 variables taken when the patient arrived at the emergency department: age, sex, level of triage, initial disposition, first diagnosis, diagnostic test and medication, and we performed a logistic regression.

Results. We included 2,476 visits of which 114 (4.6\%) were admitted. A significant direct correlation was seen between: age $>65$ years old (odds ratio[OR $]=2.1$, confidence interval [CI] 95\%,1.3-3.2; $\mathrm{p}=0.001)$; male sex (OR=1.6, IC 95\%,1.1-2.4 $\mathrm{p}=0.020$ ); dyspnea (OR=5.2, IC $95 \%, 2.8-9.7 ; \mathrm{p}<0.0001)$, abdominal pain ( $\mathrm{OR}=4.7$, IC $95 \%, 2.7-8.3 ; \mathrm{p}<0.0001)$; acute care initial disposition (OR=8.9, IC 95\%, 5.4-14.9; $\mathrm{p}<0.0001$ ), diagnostic test (OR=1.1, IC 95\%,0.9-1.3; $\mathrm{p}=0.064)$ and treatment prescription (OR=2.6, IC95\%,1.6-4.2; $\mathrm{p}=<0.0001)$. The model had a sensitivity of $76 \%$ and a specificity of $82 \%$ (area under curve 0.85 [IC $95 \% 0.81-0.88 ; \mathrm{p}<0.001$ ]).

Conclusions. The in-hospital admission prediction model is a good and useful tool for predicting the in-hospital beds needed when patients arrive at the emergency department.

Key words. Triage. Emergency department. Admission. Saturation.
1. Servicio Admisión y Documentación Clínica. Hospital Clínico San Carlos. Madrid.

2. Servicio de Medicina Preventiva e Instituto de Investigación Sanitaria del Hospital Clínico San Carlos. Madrid.

3. Servicio de Urgencias. Hospital Clínico San Carlos. Madrid.

4. Universidad Camilo José Cela. Madrid.

\section{Correspondencia:}

Dr. Francisco Javier Martín-Sánchez

Hospital Clínico San Carlos

Servicio de Urgencias

C/ Profesor Martín Lagos, s/n

28040 Madrid

E-mail: fjjms@hotmail.com

Recepción: 19 de enero de 2012

Aceptación provisional: 14 de febrero de 2012

Aceptación definitiva: 27 de febrero de 2012 


\section{INTRODUCCIÓN}

La saturación de los servicios de urgencias hospitalarios (SUH) es una situación extendida en un gran número de países que se asocia a baja calidad asistencial. Existen estudios que han correlacionado la saturación de los SUH con el manejo inadecuado del dolor ${ }^{1}$, el retraso en el tiempo de administración del antibiótico ${ }^{2-4}$, el elevado riesgo de infección por medidas deficientes de aislamiento $^{5}$, la mayor probabilidad de irse de alta sin valoración médica, así como una mayor mortalidad a corto plazo ${ }^{6,7}$.

El desequilibrio entre la demanda y los recursos aportados por el hospital ocasiona la permanencia excesiva en el servicio de Urgencias de los pacientes con orden de ingreso de hospitalización, siendo ésta una de las principales causas evitables de saturación de los $\mathrm{SUH}^{1,8}$. Se ha descrito que el retraso del ingreso hospitalario de estos pacientes se asocia con un incremento de los efectos adversos $^{9,10}$, la mortalidad a corto plazo, la estancia media intrahospitalaria y los costes asociados $^{7,11}$. Además, dicha situación reduce la capacidad de los $\mathrm{SUH}$, restringiendo la correcta atención de nuevos pacientes.

La reducción del tiempo de estancia en los SUH pendiente de cama de hospitalización se ha convertido en uno de los retos de la gestión hospitalaria. De hecho, se han publicado diversas estrategias con el fin de reestructurar el sistema de ingreso hospitalario, ya que permitiría disminuir el número de pacientes pendientes de ingreso y trabajar así de forma más eficiente con un nivel de riesgo aceptable para los pacientes ${ }^{12}$. Las más significativas han sido: simplificar el procedimiento de ingreso ${ }^{13}$, incluir médicos en el proceso de admisión tanto de la planta de hospitalización ${ }^{14}$ como del servicio de Urgencias ${ }^{15}$, mejorar el proceso de altas precoces hospitalarias ${ }^{16}$ o la creación de unidades de corta estancia dependientes de los $\mathrm{SUH}^{8,12,17,18}$.

En la actualidad, dados los tiempos empleados desde la llegada del paciente al SUH hasta la comunicación de la necesidad de ingreso así como la franja horaria de los picos de frecuentación de los pacientes ${ }^{19}$, hace difícil al servicio de admisión el conocimiento sobre la necesidad real de camas hospitalarias y la toma de decisiones en ese mismo día. En este sentido, Miró y col desarrollaron una estimación de la provisión de camas de hospitalización basándose en indicadores indirectos como la actividad asistencial a primera hora de la mañana ${ }^{20}$. Por tanto uno de los mayores retos a la hora de gestionar los ingresos urgentes consiste en que el servicio de Admisión conozca de forma precoz la necesidad de cama de hospitalización, lo cual permitiría tomar medidas de gestión casi a tiempo real.

La mayoría de los autores han intentado calcular la probabilidad de ingreso hospitalario basándose en variables individuales, como las subjetivas del médico y/o enfermera $^{21,22}$, las hemodinámicas ${ }^{23}$ o los niveles de gravedad de los sistemas de tria$\mathrm{je}^{24}$ demostrando una capacidad limitada.

Por tanto, el objetivo de nuestro trabajo fue desarrollar un modelo de predicción de ingreso hospitalario a la llegada del paciente al SUH, con el fin de conocer la necesidad de camas hospitalarias casi a tiempo real, y así preveer los recursos asistenciales necesarios de forma precoz.

\section{MATERIAL Y MÉTODOS}

Se ha realizado este estudio en el hospital Clínico San Carlos (HCSC), hospital terciario universitario ubicado en Madrid, con una población de referencia aproximada de 500.000 personas, una media de atenciones urgentes de 500 diarias y un número total de ingresos urgentes cercano a 20.000 anualmente.

El SUH se dividía en el momento del estudio en: área de clasificación, sala de reanimación, sala de agudos, área de pacientes ambulantes que incluía a ciertas especialidades (traumatología, pediatría, psiquiatría, ginecología y obstetricia, oftalmología, otorrinolaringología y sala de curas), sala de observación y unidad de corta estancia.

Se seleccionaron todos los pacientes consecutivos filiados para el triaje del SUH del HCSC durante el periodo del 1 al 30 de noviembre de 2007 entre las 8-22 horas.

\section{Diseño del estudio}

Se realizó un estudio observacional de cohorte prospectivo con todos los pacien- 
tes filiados para el triaje durante el periodo del estudio. Los pacientes eran atendidos por una enfermera experta que los clasificaba según su motivo de consulta en diferentes niveles de gravedad según el Sistema de Triaje de Manchester (STM).

El STM es una escala de triaje que contempla 52 motivos de consulta y que convierte el concepto de síntoma centinela en categoría de presentación e introduce el concepto de discriminante clave para determinar el nivel de clasificación. Distingue 5 niveles de clasificación, adjudicando unos tiempos de espera máximos de atención según el nivel: rojo (emergencia o nivel I) implica la necesidad de asistencia inmediata, naranja (muy urgente o nivel II) en 10 minutos, amarillo (urgente o nivel III) en 60 minutos, verde (estándar o nivel IV) en 120 minutos, azul (no urgente o nivel V) en 240 minutos $^{25}$.

Los pacientes clasificados como urgentes (rojo, naranja o amarillo) fueron valorados por un médico adjunto (facultativo de triaje) que decidía la ubicación inmediata (sala de agudos o zona ambulante) y la solicitud de pruebas complementarias y prescripción de tratamiento a la llegada del paciente; los no urgentes (verde o azul) fueron ubicados, sin valoración previa por el médico, directamente en la zona ambulante. Los pacientes ubicados en la sala de agudos fueron aquellos potencialmente definidos como de alta complejidad. El criterio de ubicación inmediata en dicho nivel asistencial era, a juicio del facultativo, en base a unos criterios médicos generales (alteración del nivel de conciencia, constantes vitales, saturación basal de oxígeno) o específicos del síntoma de consulta (ver material suplementario).

Los pacientes filiados para el triaje en el momento del estudio eran aquellos que acudían estables exceptuando algunas especialidades (traumatología, ginecología y obstetricia, pediatría, oftalmología, otorrinolaringología y psiquiatría). Los pacientes con inestabilidad respiratoria y/o hemodinámica o que llegaban en ambulancia medicalizada eran ubicados inicialmente de forma inmediata en la sala de agudos.

A la llegada del paciente al SUH se recogieron las siguientes variables independientes: grupo edad ( $\leq 65$ ó > 65 años), sexo (hombre o mujer), nivel de gravedad según el STM (rojo, naranja, amarillo, verde o azul), ubicación inicial (sala de agudos o zona de atención ambulante, diagnóstico de entrada (motivo de consulta entre las 52 categorías del STM), solicitud de al menos una prueba complementarias como laboratorio, radiología o electrocardiograma (sí o no) y prescripción de al menos un medicación por vía parenteral (sí o no).

Como variable dependiente o de resultado se seleccionó la ubicación final (ingreso o alta). La ubicación final era definida por los médicos adjuntos del servicio responsables de cada área asistencial, basándose en las recomendaciones de ingreso de los protocolos del SUH del HCSC (Tabla 1). Dicho colectivo era desconocedor de la realización del estudio y rotaba por las diferentes unidades asistenciales.

Las variables cuantitativas analizadas se expresaron como media (desviación estándar) o como mediana (rango intercuartílico) en caso de asimetría, y las variables cualitativas se expresaron como frecuencias. Se evaluó la asociación entre variables cualitativas con el test de $\chi^{2}$ o prueba exacta de Fisher. Se realizaron comparaciones ad hoc, dos a dos, por el método de Bonferroni. Se estableció una estrategia de construcción del modelo de regresión logística para evaluar la probabilidad de ingreso hospitalario a partir de las variables que en el análisis univariado presentaron $\mathrm{p}<0,05$. Al ser un modelo con intención predictiva se construyó el modelo saturado o completo y se obtuvo la sensibilidad y la especificidad. Se optó por el modelo más parsimonioso (menos parámetros) y más predictivo (sensibilidad y especificidad mayor). Se construyeron las curvas de rendimiento diagnóstico (COR) de los modelos ajustados. Se presentan las áreas bajo la curva y su intervalo de confianza al $95 \%$. Se presentan las "odds ratios" y sus intervalos de confianza al 95\% (IC 95\%). Se calcularon las probabilidades predichas y se estratificaron por las características de la población estudiada. En todos los contrastes de hipótesis se rechazó la hipótesis nula con error $\alpha$ menor a 0,05 . El análisis estadístico se llevó a cabo con la ayuda del paquete estadístico SPSS $15.0^{\circledR}$. 
Tabla 1. Criterios de ubicación inmediata en sala de agudos por síntoma de consulta

\begin{tabular}{||l|}
\hline GENERAL \\
1.- Alteración del nivel de conciencia. \\
2.- Procedimientos técnicos. \\
3.- Trastorno de la movilidad significativo. \\
4.- Inestabilidad hemodinámica. \\
5.- Insuficiencia respiratoria. \\
6.- Alteraciones significativas en el electrocardiograma. \\
\end{tabular}

\section{DOLOR TORÁCICO}

1.- Antecedentes de cardiopatía isquémica o factores de riesgo cardiovascular.

2.- Clínica típica de cardiopatía isquémica.

3.- Insuficiencia respiratoria.

4- Alteraciones signiticativas en el electrocardiograma.

\section{HEMORRAGIA DIGESTIVA}

1.- Edad mayor de 75 años.

2.- Discrasia sanguínea 0 anticoagulación.

3.- Enfermedad concomitante: hepatopatía, neoplasia digestiva, etc...

4.- Cirugía o técnica invasiva (endoscopia) reciente.

5. - Tacto rectal: melenas orectorragia con coágulos.

\section{DOLOR ABDOMINAL}

1.- Comienzo súbito y gran intensidad

2.- Dolor abdominal con wómitos repetidos.

3.- Dolor abdominal con sangrado rectal, vaginal o hematemesis

4.- Traumatismo abdominal de intensidad media alta o penetrante.

\section{CEFALEA}

1.- Comienzo súbito y alta intensidad.

2. - Cefalea desencadenada exclusivamente por la tos o el estuerzo.

3.- Fiebre y rigidez de nuca.

4.- Alteraciones en el comportamiento.

5.- Focalidad neurológica.

6. - Crisis convulsiva.

\section{FIEBRE}

1.- Temperatura? $41^{\circ} \mathrm{C}$

2.- Rápido e intenso deterioro del estado general.

3.- Signos de hipoperfusión periférica.

4.- Alteración del nivel de conciencia.

5. - Acompañada de signos meníngeos

6.- Crisis convulsiva o antecedentes de epilepsia.

7.- Exantema purpúrico

\section{INTOXICACIONES}

1.- Bajo nivel de conciencia

2.- Insuficiencia ventilatoria.

3.- Focalidad neurológica.

4.- Necesidad de lavado gástrico (ingesta $<1$ hora y bxico potencialmente letal)

5. - Sospecha de tenómeno comicial previo.

6.- Fármacos que precisen monitorizacín cardiaca.

7.- Sospecha de tóxico latente en paciente asintomático.

8.- Utilización de antídotos potencialmente iatrogénicos.

\section{PÉRDIDA DE CONOCIMIENTO}

1.- $E$ dad $>$ de 60 años.

2.- Antecedentes de insuficiencia cardiaca o arritmia ventricular

3.- Historía de enfermedad coronaria.

4.- Asociado a dolor torácico.

5. - Alteración significativa en el electrocardiograma

6. - Sospecha de entermedad valvular

7.- Enfermedad congénita cardiaca y/o antecedentes familiares de muerte subita.

8. - Sincope de esfuerzo en paciente joven.

\section{DISNEA}

1.- Alteración del nivel de conciencia.

2.- Incapacidad para hablar, uso de músculos accesorios o respiración paradójica

3.- Dolor torácico típico de cardiopatía isquémica.

4.- Saturación de oxígeno menor del $90 \%$.

5. - Saturación de oxígeno menor del $95 \%$ con al menos dos de las siguientes: frecuencia respiratoria $>25 \mathrm{rpm}$, temperatura $>37,5^{\circ} \mathrm{C}$ o antecedentes de EPOC.

6. - Frecuencia cardiaca $<60 \mathrm{lat} / \mathrm{min} 0>120 \mathrm{lat} / \mathrm{min}$

\section{TRAUMATISMO COSTAL}

1.- Traumatismos de alta intensidad.

2.- Insuticiencia respiratoria

3. - Dolor a la palpación en primeras costillas

4.-Alteraciones significativas en el electrocardiograma.

5.- Alteraciones en el murmullo vesicular.

7.- Enfermedad pulmonar conocida.

8.- Politraumatizados.

TRAUMATISMO CRANEOENCEFÁLICO

1.- Glasgow < 15 puntos.

2.- Pérdida de conocimiento.

3.- Náuseas o vómitos recientes.

4.- Amnesia del episodio.

5. - Focalidad neurológica.

6.- Discrasia sanguínea o anticoagulación.

7.- Paciente intoxicado

8. - Traumatismo de alta intensidad.

\section{REACCIÓN ALÉRGICA}

1.- Dificultad respiratoria.

2.- Sibilancias

3.- Edema de úvula.

4.- Palpitaciones o dolor torácico

5.- Síntomas gastroinestinales.

6. - Mareo y/o vértigo

7.- Pérdida de conocimiento

8.- Secundario a IECAs o deficiencia de C1 inhibidor 


\section{RESULTADOS}

Se incluyeron un total de 2.476 episodios con una mediana de edad de 50,3 años (rango 15-108), con un predominio del sexo femenino (1.383 [55,8\%]). En cuanto al diagnóstico de entrada, el más frecuente fue el adulto con malestar general (436[17,6\%]), seguido del paciente con dolor abdominal (342[13,8\%]), problemas de extremidades $(268[10,8 \%])$, proble- mas urinarios $(217[8,8 \%])$, dolor torácico $(163[6,6 \%])$, y disnea $(150[6,1 \%])$. Los niveles de gravedad según el STM fueron: 0 rojos ( $0 \%), 64$ naranjas (2,6\%), 798 amarillos (32,2\%), 1.525 verdes $(61,6 \%), 89$ azules $(3,6 \%)$. Del total de los pacientes, 194 $(7,8 \%)$ fueron ubicados inicialmente en la sala de agudos y $2.282(92,2 \%)$ en la zona ambulante (Fig. 1). El porcentaje de ingreso fue del $4,6 \%$.

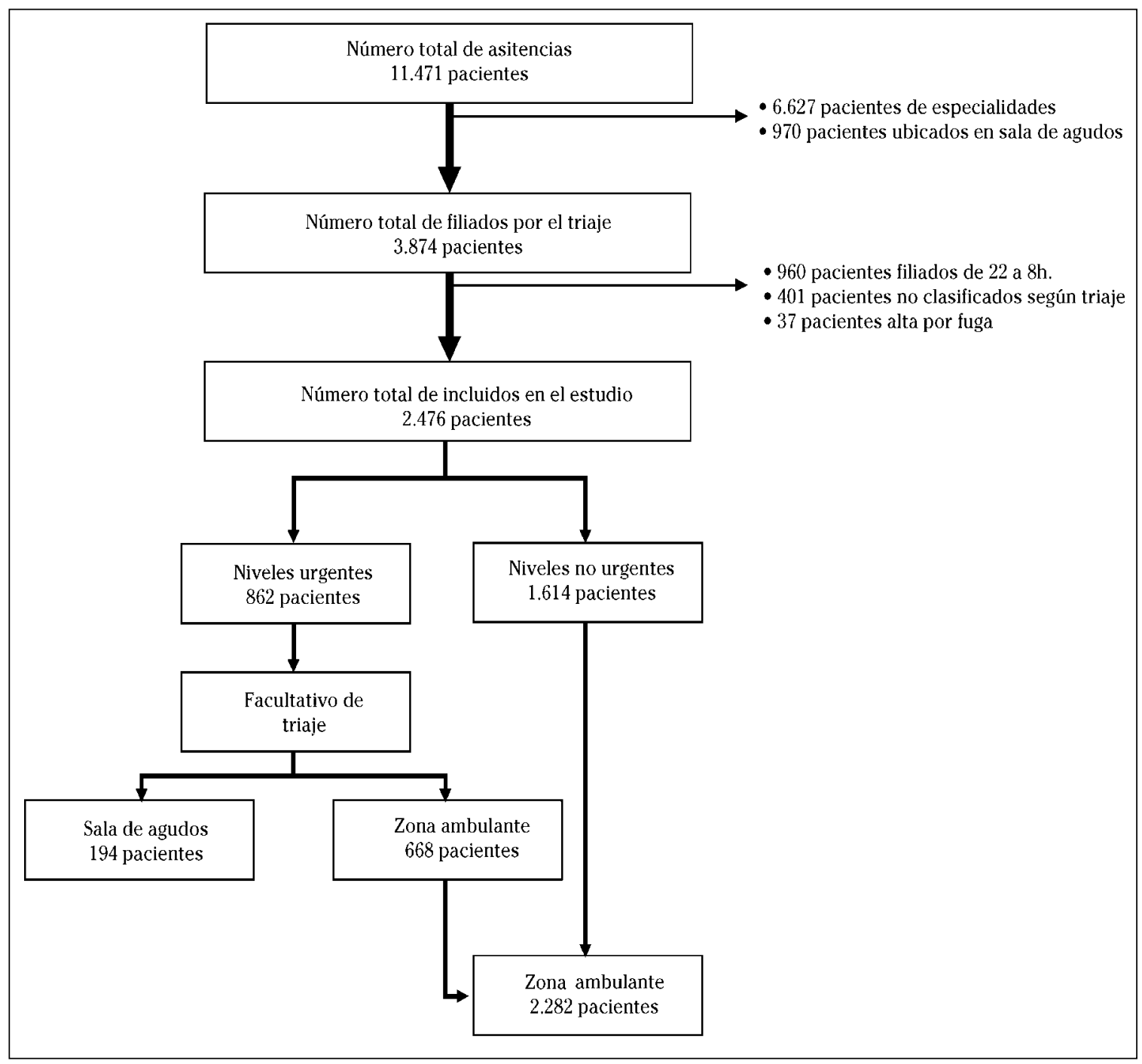

Figura 1. Pacientes incluidos en el análisis del estudio. 
El estudio univariable demostró como variables estadísticamente significativas asociadas con el ingreso hospitalario: el grupo de edad > 65 años, el sexo masculino, el diagnóstico sindrómico de entrada, el nivel de gravedad según STM, la ubicación inicial determinada por el facultativo de triaje, y la solicitud de pruebas complementarias y la prescripción de tratamiento (Tabla 2).

Tabla 2. Análisis univariable del efecto de las variables independientes en el ingreso hospitalario

\begin{tabular}{|c|c|c|}
\hline Variable & Ingreso n (\%) & $\mathbf{P}$ \\
\hline Grupo de edad > 65 años & & $<0,0001^{*}$ \\
\hline Sí & $66(8,6)$ & \\
\hline No & $49(2,9)$ & \\
\hline Sexo masculino & & $0,001^{*}$ \\
\hline Sí & $69(6,2)$ & \\
\hline No & $47(3,4)$ & \\
\hline Diagnóstico de entrada & & $<0,0001^{*}$ \\
\hline Adulto con malestar general & $18(4,1)$ & \\
\hline Disnea & $30(20)$ & \\
\hline Dolor abdominal & $29(8,5)$ & \\
\hline Dolor torácico & $9(5,5)$ & \\
\hline Nivel de gravedad del triaje & & $<0,0001 *$ * \\
\hline Naranja & $14(21,9)$ & \\
\hline Amarillo & $64(8,0)$ & \\
\hline Verde & $37(2,4)$ & \\
\hline Azul & $0(0)$ & \\
\hline Ubicación inicial & & $<0,0001$ * \\
\hline Sala de agudos & $46(23,7)$ & \\
\hline Zona de atención ambulante & $68(3)$ & \\
\hline Solicitud de pruebas complementarias & & $<0,0001$ * \\
\hline Sí & $76(6,2)$ & \\
\hline No & $39(3,1)$ & \\
\hline Prescripción de tratamiento parenteral & & $0,020^{*}$ \\
\hline Sí & $47(6,1)$ & \\
\hline No & $68(4,0)$ & \\
\hline
\end{tabular}

*Test de la $\chi^{2} ;{ }^{* *} \chi^{2}$ de tendencia lineal.

Tras el análisis multivariable mantuvieron la significación estadística: edad mayor de 65 años, sexo masculino, diagnóstico sindrómico de entrada, la ubicación inicial sala de agudos, y la solicitud pruebas complementarias y la prescripción de tratamiento (Tabla 3).

$\mathrm{Al}$ eliminarse la variable del nivel de gravedad del triaje según el STM, se obtuvo un modelo final que incluyó 9 variables independientes: ubicación inicial en sala de agudos, sexo masculino, edad mayor de 65 años, diagnóstico de entrada (adulto con malestar general, disnea, dolor abdominal o dolor torácico), y la solicitud de al menos una prueba complementaria y prescripción de al menos un tratamiento (área bajo la curva fue 0,85 [IC 95\%; 0,81$0,88 ; \mathrm{p}<0,001])$. El modelo tenía una sensibilidad del $75,5 \%$ y una especificidad del $81,5 \%$ de predicción de ingreso hospitalario. 
Tabla 3. Estudio multivariable del efecto de las diferentes variables independientes en el ingreso hospitalario

\begin{tabular}{lccc}
\hline & $\begin{array}{c}\text { Coeficiente cálculo } \\
\text { probabilidad }\end{array}$ & OR (IC95\%) & P \\
\hline Grupo de edad (> 65 años) & 0,7 & $2,1(1,3-3,2)$ & 0,001 \\
Sexo (masculino) & 0,5 & $1,6(1,1-2,4)$ & 0,020 \\
Diagnóstico de entrada & 0,5 & $1,7(0,9-3,2)$ & $<0,0001$ \\
Adulto con malestar general & 1,7 & $5,2(2,8-9,7)$ & 0,107 \\
Disnea & 1,7 & $4,7(2,7-8,3)$ & $<0,0001$ \\
Dolor abdominal & 0,4 & $1,5(0,6-3,4)$ & $<0,0001$ \\
Dolor torácico & 2,2 & $8,9(5,4-14,9)$ & 0,364 \\
Ubicación inicial (sala de agudos) & 1 & $2,6(1,6-4,2)$ & $<0,0001$ \\
Medicaciones (sí) & 0,1 & $1,1(0,9-1,3)$ & $<0,0001$ \\
Pruebas complementarias (sí) & & 0,064 \\
\hline
\end{tabular}

${ }^{*}$ La constante para el cálculo de probabilidad de ingreso es igual a 5,2.

A partir del presente modelo se puede construir una fórmula matemática con los coeficientes de cada variable para calcular la probabilidad de ingreso de cada paciente de forma individualiza (Tabla 3). Por ejemplo, un hombre mayor de 65 años que acude con disnea, se ubica en la sala de agudos, se le solicita analítica y radiografía y se pauta medicación parenteral con esteroides, y tendría una probabilidad de ingreso del $89 \%$. Por otro lado, una mujer de 50 años que consulta a urgencias por malestar general y se ubica inicialmente en la zona ambulante sin solicitud de ninguna prueba ni prescripción de tratamiento, tendría una la posibilidad de ingreso del $1,8 \%$.

\section{DISCUSIÓN}

El presente trabajo ha desarrollado un modelo matemático derivado de la casuística real al conocimiento apriorístico de la necesidad de camas para ingresos desde el servicio de Urgencias para un momento determinado. Dicho modelo contiene variables demográficas, como edad y género, y clínicas, como el motivo de consulta descrito por el triaje (adulto con malestar general, dolor abdominal, disnea y dolor torácico), la solicitud de pruebas complementarias y prescripción de fármacos, y la ubicación inicial determinada por un médico del servicio tras la primera valoración del paciente, y es aplicable a los adultos que consultaron por un problema médico y que se encontraban estables a su llegada a urgencias. Es un modelo predictivo de ingreso hospitalario con buena capacidad discriminadora (área bajo la curva de 0,85 ) por lo que podría ser una estrategia válida a la hora de planificar los recursos a destinar a los ingresos urgentes. Además a partir de dicho modelo, se puede aplicar una fórmula matemática con los coeficientes de cada variable, que permite calcular la probabilidad de ingreso de cada paciente de forma individualiza (sensibilidad del 75,5\% y especificidad del 81,5\%).

Para mostrar la transcendencia clínica que puede tener el presente modelo, nos gustaría destacar que en el año de realización del presente trabajo, nuestro centro tenía una mediana de tiempo desde la llegada del paciente a urgencias hasta la comunicación de ingreso de 5 horas (RIC $3 \mathrm{~h}$ 23 min-7h $38 \mathrm{~min}$ ). Por tanto, el disponer 
de un modelo de predicción de ingreso a la llegada del paciente a urgencias es de vital importancia, ya que permitiría anticipar la necesidad de camas hospitalarias para ingreso urgente, y así poder reorganizar los ingresos programados casi "a tiempo real».

Un trabajo previo intentó desarrollar un modelo de predicción de ingreso en población americana a través de las bases de datos administrativas ${ }^{26}$, e incluyó variables como: edad (en deciles), hora de registro (periodos de 4 horas), modo de llegada (ambulancia, helicóptero, coche, caminando, otro), queja principal codificada ICD-9 (172 códigos), el nivel de gravedad (5 categorías según Emergency Severity Index), tipo de consulta (24 categorías), la presencia de al menos una prueba complementarias de laboratorio (si/no), de radiología (si/no) y un electrocardiograma (si/no). Dicho modelo presentó una alta sensibilidad $95 \%$ pero limitada especificidad $62 \%^{27}$.

El porcentaje de ingreso hospitalario en nuestro estudio fue del 4,6\%. Dicho porcentaje, teniendo en cuenta que se trata de un hospital terciario universitario, es significativamente inferior al $10 \%$ que se considera según los estándares de calidad ${ }^{19}$. Una posible justificación podría ser que el presente trabajo incluyó a una población seleccionada, es decir, se excluyeron los pacientes inestables o que fueron trasladados en unidad móvil de emergencia "UVI móvil" así como determinadas especialidades descritas anteriormente.

En nuestro estudio, fue la ubicación inicial "sala de agudos" por parte de un facultativo del servicio a la llegada del paciente la variable independiente que más condicionó la probabilidad de ingreso hospitalario. De hecho, un paciente ubicado de forma inicial en la zona ambulante tiene una probabilidad de alta cercana al $97 \%$, y si se destina en la sala de agudos tiene una probabilidad de ingreso del $24 \%$. Otros autores ya han descrito la necesidad de un médico como apoyo al triaje de enfermería para la detección de pacientes de alta complejidad, y así poder ubicarlos adecuadamente a su llegada a un SUH, demostrando también su asociación con el ingreso hospitalario $^{28}$.
Respecto las variables demográficas, anciano y sexo masculino ya habían sido descritas previamente por otros autores como predictoras de ingreso ${ }^{29,30}$, aunque sobre esta última existe una falta de unanimidad en la literatura ${ }^{31}$. La variable del diagnóstico de entrada según el STM, y más específicamente disnea, dolor abdominal, dolor torácico y adulto con malestar general, son síndromes que se asocian a una valoración más exhaustiva, y por tanto posiblemente a mayor probabilidad de ingreso. De hecho las variables pruebas complementarias y administración de tratamiento son también representativas de este punto ${ }^{27}$.

Estudios previos han demostrado la importancia del nivel de gravedad del triaje ${ }^{24}$, y de las variables hemodinámicas ${ }^{23}$ en la predicción de ingreso hospitalario. Respecto a las variables hemodinámicas, nuestro estudio no las incluyó de manera aislada ya que el STM las incluye como discriminadores clínicos.

Es curioso que en nuestro trabajo, a pesar de que los niveles de gravedad según el STM se asociaban de forma directamente proporcional con el porcentaje de ingreso hospitalario, no influyeron a la hora de predecir el ingreso hospitalario. Trabajos previos han demostrado que los sistemas de triaje de cinco categorías predicen el ingreso hospitalario, así como la existencia de una correlación entre el nivel de prioridad de atención y la mortalidad ${ }^{24}$. Por otra parte, es conocido que el STM tiene importantes limitaciones a la hora de predecir ingresos ya que usa exclusivamente discriminadores clínicos, como por ejemplo el dolor severo que clasifica al paciente con un alto nivel de gravedad, aunque no sea necesaria la hospitalización. Otro sistema de triaje de cinco categorías, como el Emergency Severity Index ${ }^{32}$, ha demostrado ser un sistema de triaje más predictivo a la hora del ingreso hospitalario que el STM, probablemente porque valora la necesidad de recursos ${ }^{24}$. En esta línea, trabajos previos han documentado la incapacidad del STM a la hora de predecir el ingreso, debido a que la complejidad del paciente no depende exclusivamente del nivel de 
urgencia del triaje, sino de otras variables, como la edad, el motivo de consulta, la comorbilidad, la situación basal funcional y cognitiva, y la necesidad de recursos ${ }^{28,33}$. De hecho, nuestro grupo de trabajo aboga por la necesidad de un médico como apoyo al triaje, principalmente en los casos de niveles de gravedad clasificados como urgentes, para detectar los pacientes de alta complejidad $^{28}$.

Referente a las limitaciones del estudio, en primer lugar, la población del estudio no englobó a todos los episodios, sino sólo a aquellos que comprendían a pacientes adultos que se encontraban estables a su llegada al SUH y que previamente a su ubicación inicial habían sido clasificados por un sistema de triaje durante dicho periodo. Dicha selección de pacientes fue consecuencia de los circuitos asistenciales de nuestro centro, así como la restricción a dicho intervalo de tiempo a la posibilidad de disponer de un facultativo de apoyo al triaje. Por lo tanto, las conclusiones no pueden ser generalizadas a cualquier paciente, patología, periodo de tiempo o centro hospitalario. En segundo lugar, la variable dependiente ingreso hospitalario fue a criterio del médico responsable de cada área del SUH. A pesar de ser una variable subjetiva, se basó en las guías clínicas de nuestro centro, y los médicos que tomaron las decisiones de la ubicación final durante el periodo del estudio desconocían el desarrollo del mismo y rotaron por las distintas unidades asistenciales. En tercer lugar, respecto a las variables independientes incluidas en el modelo, no se han realizado estudios de concordancia entre enfermeras para interpretar el motivo principal de consulta según el STM, ni tampoco entre médicos a la hora de la decisión de la ubicación inicial y la solicitud de pruebas complementarias y administración de tratamiento de forma inmediata. Bien es cierto, que las enfermeras han recibido en nuestro centro un curso de formación del $\mathrm{STM}^{34}$, y que los criterios de ubicación inicial estaban previamente definidos por problemas. Además la solicitud de pruebas complementarias y administración de tratamientos se basaba en los protocolos de actuación de nuestro centro. En cuarto lugar, no existe una validación del modelo de predicción de ingreso hospitalario descrito. Un problema para la realización del mismo fue la dificultad logística y el coste del procedimiento, ya que es necesario un médico de apoyo al triaje las 24 horas del día los 365 días del año.

La conclusión es que existe un posible modelo de predicción de ingreso hospitalario a la llegada del paciente al SUH, pero que son necesarios futuros trabajos ${ }^{35}$ que incluyan un mayor número de pacientes, que incluyan todo episodio que acude a urgencias, indistintamente de la edad o motivo de consulta, distintos sistemas de clasificación, y variables demográficas, clínicas y de consumo de recursos, así como un mayor periodo de observación y distintos centros, para conocer la verdadera utilidad del presente modelo de predicción de ingreso hospitalario a la llegada de los pacientes a un SUH.

\section{Agradecimientos}

A los médicos y enfermeras del Servicio de Urgencias del hospital Clínico San Carlos que han colaborado en el desarrollo del estudio

\section{BIBLIOGRAFÍA}

1. Flores CR. La saturación de los servicios de urgencias: una llamada a la unidad. Emergencias 2011; 23: 59-64.

2. Pines JM, Hollander JE, Localio AR, Metlay JP. The association between Emergency Department crowding and hospital performance on antibiotic timing for pneumonia and percutaneous intervention for myocardial infarction. Acad Emerg Med 2006; 13:873-878.

3. McCAIG LF, NAWAR EW. National hospital ambulatory medical care survey: 2004 emergency department summary. Adv Data 2006; 372: $1-29$.

4. Fee C, Weber EJ, MaAk CA, Bacchetti P. Effect of emergency department crowding on time to antibiotics in patients admitted with community-acquired pneumonia. Ann Emerg Med 2007; 50: 501-509. 
5. Moran GJ, Krishnadasan A, Gorwitz RJ,Fosheim GE, Mc dougal K, CAREY RB et al. Methicillinresistant $\mathrm{S}$. aureus infections among patients in the emergency department. N Engl J Med 2006; 355: 666-674.

6. RICHARDSON DB. Increase in patient mortality at 10 days associated with emergency department overcrowding. Med J Aust 2006; 184: 213-216.

7. Guttmann A, Schull MJ, Vermeulen MJ, Stukel TA. Association between waiting times and short term mortality and hospital admission after departure from emergency department: population based cohort study from Ontario, Canada. BMJ 2011; 342: d2983.

8. Juan A, Enjamio E, Moya C, García Fortea c, Castellanos J, Ramón Pérez J et al. Impacto de la implementación de medidas de gestión hospitalaria para aumentar la eficiencia en la gestión de camas y disminuir la saturación del servicio de urgencias. Emergencias 2010; 22: 249-253.

9. Tomás S, Chanovas M, Roqueta F, Alcaraz J, ToRANZO T, y GRUPO DE TRABAJO EVADUR-SEMES. EVADUR: eventos adversos ligados a la asistencia en los servicios de urgencias de hospitales españoles. Emergencias 2010; 22: 415428.

10. Bañeres J. La seguridad en los servicios de urgencias. Emergencias 2010; 22: 81-82.

11. Huang Q, Thind A, Dreyer JF, Zaric GS. The impact of delays to admission from the emergency department on inpatient outcomes. BMC Emerg Med 2010; 10:16.

12. Moloney ED, Bennett K, O'Riordan D, Silke B. Emergency department census of patients awaiting admission following reorganisation of an admissions process. Emerg Med J 2006; 23: 363-367.

13. Amarasingham $R$, Swanson TS, Treichler DB, Amarasingham SN, ReEd WG. A rapid admission protocol to reduce emergency department boarding times. Qual Saf Health Care 2010; 19: 200-204.

14. Howell EE, Bessman ES, Rubin HR. Hospitalists and an innovative emergency department admission process. J Gen Intern Med 2004; 19: 266-268.

15. Brun Romero FM, Benítez Macías JF, García GiL D, López Álvaro J. Tiempo de demora para la hospitalización tras la implantación del ingreso directo a cargo del Servicio de Urgencias. Rev Clin Esp 2010; 210: 159-162.

16. García Alonso D, Enguix N, Valverde L, Castells M, Pascual I, EzQuerda a et al. Resultado de un proceso para la mejora de las altas hospitalarias precoces. Emergencias 2011; 23: 29-34.

17. Ovens H. Saturación de los servicios de urgencias. Una propuesta desde el Sistema para un problema del Sistema. Emergencias 2010; 22: 244-246.

18. Palacios G, Emparan García de Salazar C. Impacto económico de la unidad de hospitalización de urgencias. Emergencias 2010; 22: 254-258.

19. Ministerio de Sanidad y Política Social. Unidad de urgencias hospitalarias. Estándares y Recomendaciones 2010. Disponible en: www. msc.es//organizacion/sns/planCalidadSNS/ docs/UHH.pdf

20. Miró O, Salgado E, Bragulat E, Junyent M, AsenJo MA, SÁnchez M. Estimación de La actividad en urgencias y su relación con la provisión de camas de hospitalización. Med Clin (Barc) 2006; 127: 86-89.

21. Dent AW, Weiland TJ, Vallender L, Oettel NE. Can medical admission and length of stay be accurately predicted by emergency staff, patients or relatives? Aust Health Rev 2007; 31: 633-641.

22. Beardsell I, Robinson S. Can emergency department nurses performing triage predict the need for admission? Emerg Med J 2011; 28: 959-962.

23. Burch VC, TARr G, Morroni C. Modified early warning score predicts the need for hospital admission and inhospital mortality. Emerg Med J 2008; 25: 674-678.

24. Van der Wulp I, SchriJvers AJ, Van Stel HF. Predicting admission and mortality with the Emergency Severity Index and the Manchester Triage System: a retrospective observational study. Emerg Med J 2009; 26: 506-509.

25. Coоke MW, Jinks S. Does the Manchester triage system detect the critically ill? J Accid Emerg Med 1999; 16: 179-181.

26. Vaillancourt SD, Schull MJ. ¿Puede la investigación con bases de datos administrativas ayudarnos a proporcionar una mejor atención a nuestros pacientes? Emergencias 2011; 23: 329-332.

27. Leegon J, Jones I, Lanaghan K, Aronsky D. Predicting hospital admission for emergency department patients using a bayesian network. AMIA Annu Symp Proc 2005:1022.

28. Martín SÁnchez FJ, GonzÁlez del Castillo J, ZAmorano J, Candel FJ, Armengol J, Villarroel P et al. El facultativo, un elemento necesario en el triaje de un Servicio de Urgencias en 
un hospital terciario. Emergencias 2008; 20: 41-44.

29. WALKER LL. Inpatient and emergency department utilization: the effect of distance, social class, age, sex, and marital status. JACEP 1976; 5: 105-110.

30. Blatchford O, Capewell S, Murray S, BlatCHFORD M. Emergency medical admissions in Glasgow: general practices vary despite adjustment for age, sex, and deprivation. $\mathrm{Br} \mathrm{J}$ Gen Pract 1999; 49: 551-554.

31. Bankart MJ, BaKer R, RASHid A, Habiba M, BANERJEE J, Hsu R Y cols. Characteristics of general practices associated with emergency admission rates to hospital: a cross-sectional study. Emerg Med J 2011; 28: 558-563.
32. WUERZ R. Emergency severity index triage category is associated with six-month survival. ESI Triage Study Group. Acad Emerg Med 2001; 8: 61-64.

33. Nogué S, Ramos A, Portillo M, Bohils M. Adecuación de un sistema de triaje y del circuito asistencial en urgencias al paciente intoxicado. Emergencias 2010; 22: 338-344.

34. Escoda R, Llorens P, Martín-SÁnchez FJ, Jacob J, Pavón J, GIL C et al. Efecto de una intervención formativa en urgencias en las mejoras del manejo y el tratamiento de la insuficiencia cardiaca aguda. Emergencias 2010; 22: 331-337.

35. Rodés J. Alcanzar la excelencia clínica a través de la investigación. Emergencias 2010; 22: 241-243. 